\title{
Arzneimittelversorgung: Stabilität und Korrekturen stehen im Vordergrund
}

\section{SABINE RICHARD}

Dr. Sabine Richard leitet die Abteilung „Arznei-, Heil- und Hilfsmittel" und ist Stellvertretende Geschäftsführerin der Geschäftsführungseinheit „Versorgung“ im AOK-Bundesverband, Berlin

Nach einigen bedeutsamen Arzneimittelgesetzen in der letzten Legislaturperiode steht die Arzneimittelversorgung nicht im Zentrum der gesundheitspolitischen Agenda. Im Blickpunkt steht vor allem der Abtausch der AMNOG-Bestandsmarktbewertung gegen die pauschalen Kostensteuerungsinstrumente des GKVFinanzierungsgesetzes. Bei den Regelungen zur Arzneimittelversorgung geht es darum, das Erreichte zu bewahren und weiterzuentwickeln. Bekenntnisse zur traditionellen Distributionsstruktur sollen die unzufriedene Apothekerschaft besänftigen. Kleinteilige Interventionen finden sich ebenso wie die Absicht, einen ressortübergreifenden Dialog über den Produktions- und Forschungsstandort einzurichten. Für die Weiterentwicklung der Arzneimittelversorgung werden wenige Akzente gesetzt. Überraschend und problematisch ist die weitgehende Änderung zur Wirtschaftlichkeitsprüfung der Ärzte.

\section{Der große Deal: AMNOG- Bestandsmarkt und Fortführung des Preismoratoriums}

Die Änderung, die im Zusammenhang mit den Koalitionsverhandlungen die größte Aufmerksamkeit erzielt hat, ist die Abschaffung der Bestandsmarktbewertung im Rahmen des AMNOG-Verfahrens, verbunden mit der Fortführung des Preismoratoriums sowie der Erhöhung des gesetzlichen Herstellerrabattes von sechs auf sieben Prozent, um das geplante Einsparvolumen zu erreichen. Sowohl das Preismoratorium als auch die Anhebung des gesetzlichen Herstellerrabattes von sechs auf sechzehn Prozent sind nach den geltenden Regelungen bis Ende 2013 befristet. Insofern war der Druck, noch vor Jahresende ein Gesetzgebungsverfahren auf den Weg zu bringen, sehr hoch und der erste gesundheitspolitische Testfall für die Zusammenarbeit in der großen Koalition. Durch die Initiative der Bundestagsfraktionen ist es gelungen, zumindest das Preismoratorium über das Jahresende hinaus zu verlängern.

Die mit dem AMNOG erreichte Rationalität für den am Patientennutzen orientierten Einsatz von finanziellen Ressourcen der Gesetzlichen Krankenversicherung war ein (längst überfälliger) Meilenstein für das deutsche Gesundheitswesen. Dieser Schritt steht bei anderen Leistungen wie z.B. bei den Medizinprodukten leider noch aus.

Seit dem Inkrafttreten des AMNOG teilt sich die Arzneimittelwelt in Arz- 
neimittel, die vor dem 01.01.2011 auf dem Markt gebracht wurden (der sog. Bestandsmarkt) und die Neueinführungen, die sich seit 2011 der frühen Nutzenbewertung und den Preisverhandlungen unterziehen müssen. Mit der Abschaffung der Nutzenbewertung des Bestandsmarktes wird die Reichweite dieses neuen und durchaus beispielgebenden Verfahrens deutlich reduziert. Viele für die Versorgung wesentliche und kostenintensive Arzneimittel werden damit endgültig nicht mehr bewertet und genießen nun bis zum Ende ihrer Patentlaufzeit wesentlich größere Preisspielräume als die seit 2011 auf dem Markt gebrachten Arzneimittel.

Die vollständige Streichung der Bestandsmarktbewertung gilt auch für die laufenden Verfahren, bei denen die Nutzenbewertung bereits veranlasst wurde, für die der G-BA aber noch keinen Beschluss gefasst hat. Für die Arzneimittel des Bestandsmarktes, für die das Bewertungsverfahren mit G-BABeschluss bereits abgeschlossen ist und diese Beschlüsse bereits Bestandteil der

\section{Die Arzneimittelhersteller werden ab 2014 um \\ ca. 1 Mrd. EUR entlastet.}

Arzneimittelrichtlinie geworden sind, können auch die bereits aufgenommenen Preisverhandlungen abgeschlossen werden. Hierbei handelt es sich vor allem um die Gliptine, die in der Diabetestherapie eingesetzt werden.

Nach den Berechnungen des WIdO, die auch der G-BA für seine Bestandsmarktanalysen zugrunde gelegt hat, kann man davon ausgehen, dass die ökonomischen Effekte durch die nicht realisierten Einsparungen von Preisverhandlungen und das Nichtwissen über den patientenorientierten Zusatznutzen bis über das Jahr 2020 hinaus feststellbar sind.

Das Beispiel der neuen oralen Medikamente zu Blutverdünnung verdeutlicht dies. Diese sind in den letzten Jahren überwiegend durch Indikationserweiterungen bereits auf dem Markt befindlicher Altwirkstoffe auf den Markt gekommen. Nur der Wirkstoff Apixaban, mit Marktzutritt 2011, wurde nach dem AMNOG-Verfahren bewertet. Ergebnis war ein nur geringer Zusatznutzen, bei einem Preis, der ca. 15 mal höher ist als der Preis der zweckmäßigen Vergleichstherapie (Vitamin-K-Antagonisten) und dies bei einem nicht ganz kleinen Patientenpotenzial von ca. 1,1 Mio. Patienten. Zwei andere Wirkstoffe der gleichen Gruppe mit erheblich größerer Marktbedeutung als das bewertete Produkt bleiben nach Abschaffung der Bestandsmarktbewertung unbewertet. Ihr zusätzlicher Nutzen für die Patientenversorgung bleibt damit unbekannt. Bekannt ist nur, dass die Zulassungsbehörden in puncto Arzneimittelsicherheit bereits mehrere Warnhinweise zu Kontraindikationen veröffentlicht haben.

Im Ergebnis ist der Verzicht auf die Bestandsmarktbewertung trotz aller rechtlichen, verfahrenstechnischen und praktischen Probleme, die aufgeworfen wurden, ein schwerer Schlag. Für die $\mathrm{Zu}$ kunft ist zu hoffen, dass das AMNOGVerfahren als „lernendes System“ nicht noch weitere schmerzhafte Lektionen erdulden muss.

Im Zusammenhang mit der Abschaffung der Bestandsmarktbewertung ist auf eine weitere Unwucht aufmerksam zu machen. Eine Nutzenbewertung wird nicht durchgeführt, wenn ein Altwirkstoff eine Zulassungserweiterung für ein neues Anwendungsgebiet erhält. Neue Wirkstoffe müssen sich hingegen bei jeder

Indikationserweiterung einer erneuten Bewertung stellen. Dieses Privileg erhält mit der Neuregelung zum Bestandsmarkt eine erheblich größere Bedeutung und sollte daher entfallen.

Als Kompensation für die Mehrkosten durch die Abschaffung der Bestandsmarktbewertung war die nahtlose Fortführung des Preismoratoriums sowie die Erhöhung des gesetzlichen Herstellerrabattes von sechs auf sieben Prozent vorgesehen. Die Rabatthöhe soll 6 \% nicht unterschreiten und wird ab 2015 in direktem Zusammenhang mit der Finanzlage der gesetzlichen Krankenversicherung jährlich überprüft.

Ohne eine gesetzliche Änderung (d.h., das Preismoratorium läuft aus, der Abschlag wird auf $6 \%$ abgesenkt) wären für 2014 Mehrausgaben in Höhe von ca. 2 Mrd. EUR entstanden. Auch die nun beschlossenen und zum Teil schon auf den parlamentarischen Weg gebrachten Maßnahmen verursachen durch die deutliche Absenkung des Herstellerabschlages gegenüber 2013 noch Mehrausgaben im Arzneimittelsektor in Höhe von ca. 1 Mrd. EUR für die gesetzliche Krankenversicherung. Der Herstellerabschlag wird nach dem ersten Entwurf der Fraktionen für das 14. SGB V-Änderungsgesetz nun im ersten Quartal $20146 \%$ betragen und erst ab dem 01.04. auf die im Koalitionsvertrag vereinbarten $7 \%$ angehoben.

Neben der Aufgabe des Bestandsmarktes haben die Koalitionäre noch drei durchaus sinnvolle Detailänderungen am „lernenden System“ des AMNOGVerfahrens vorgenommen.

- Zum einen soll klargestellt werden, dass die Auffassung der Kassenseite über die Preisbildung von Arzneimitteln mit Erstattungsbeträgen nach $\mathbb{1 3 0}$ b SGB V richtig war: Der Erstattungsbetrag nach den Preisverhandlungen bildet die Basis für die Anwendung der Arzneimittelpreisverordnung (und nicht der höhere Listenpreis) und mindert daher die Handelspannen und die Umsatzsteuer des Arzneimittels. Diese Differenz kann je nach Produkt mehr als 100 EUR je Packung betragen und muss bis zum Wirksamwerden der Regelung von den Kassen aufgebracht werden, weil Hersteller und Apotheker ihre Auffassung in den Preisverzeichnissen durchgesetzt haben und damit Fakten geschaffen haben.

- Zweitens sollen in Zukunft auch Vertreter der Krankenkassen an den Verhandlungen zum Erstattungsbetrag teilnehmen, um den Versorgungsaspekt zu stärken.

- Mit der dritten Änderung soll Ausweichstrategien der Hersteller entgegengewirkt werden. Für alle Wirkstoffe, die nach 2011 auf den Markt gekommen sind, soll es nur eine Phase der freien Preisbildung geben, auch wenn ein Wirkstoff von mehreren Herstellern parallel vermarktet wird.

\section{Gesetzlicher Durchgriff bei der Substitutionsausschlussliste}

Für viele Irritationen hat in den letzten Monaten die sog. Substitutionsausschlussliste gesorgt. In $\mathbb{1 2 9}$ SGB V war seit 2012 vorgesehen, dass sich Kassen- und Apothekerseite auf Bundesebene im Rahmenvertrag über die Arzneimittelversorgung auf eine Liste von Arzneimitteln einigen können, die 
von den Apothekern nicht nach den allgemein geltenden Substitutionsvorschriften ausgetauscht werden dürfen.

Ausgangspunkt für die Thematik waren über eine Petition massiv vorgetragene Bedenken, dass die generische Substitution insbesondere in der Schmerztherapie $\mathrm{zu}$ Versorgungsproblemen führe. Die Austauschbarkeit zwischen wirkstoffgleichen Arzneimitteln wurde damit für eine große Gruppe von Arzneimitteln grundsätzlich in Frage gestellt. Relevante Belege für die vorgetragenen Probleme wurden nicht präsentiert.

Das deutsche Gesundheitswesen kennt die aut idem-Substitution bereits seit vielen Jahren. Sie war ein wichtiger Grund, dass sich in Deutschland im Gegensatz $\mathrm{zu}$ anderen Ländern ein sehr starker Generikamarkt entwickeln konnte. Der Gesetzgeber hat für die von Ärzten und Apothekern als Heilberufler zu treffende Entscheidung im Einzelfall solide Leitplanken geschaffen. Der Arzt kann die Substitution durch das bloße Ankreuzen im aut idem-Feld patientenbezogen ausschließen. Erlaubt der Arzt die Substitution, kann der Apotheker sie gleichwohl aus eigener Einschätzung unterlassen, wenn er aus seiner pharmazeutischen Sicht Bedenken dokumentiert. Insofern wären weitergehende Instrumente für die Regelung der Substitution gar nicht notwendig.

Insbesondere durch die Substitutionsverpflichtung der Apotheken gem. \129 Abs. 1 SGB V erreichten die von der GKV geschlossenen Rabattverträge ihre Bedeutung in der Versorgung. Sie erleichtert dem Arzt eine wirtschaftliche Verordnung, denn er überlässt dem Apotheker durch die Freigabe des aut-idem-Feldes auf dem Rezept die Auswahl des abzugebenden rabattierten Arzneimittels. Im August 2013 wurden ca. 60 \% der Verordnungen zu Lasten der AOK im generikafähigen Markt rabattiert abgegeben. Dabei schwankt die Umsetzungsquote zwischen den Wirkstoffen erheblich. So erreichen die Rabattprodukte im August 2013 z.B. beim Wirkstoff Pantoprazol einen Umsatzanteil von knapp $92 \%$, während der Umsatzanteil der Rabattprodukte beim Schmerzmittel Morphin nur $41,5 \%$ betrug. Hieran zeigt sich, dass Ärzte und Apotheker die Substitution sehr individuell und verantwortungsbewusst handhaben.

Seit sich die Substitution vor allem an den Rabattverträgen der Krankenkassen ausrichtet und nicht mehr an den Einkaufsbedingungen der Apotheken, wird die Substitution in der Versorgungslandschaft wieder kritischer diskutiert und auch gegenüber den Patienten kritischer begleitet. Es wird häufig übersehen, dass es in den Jahren der umfangreichen Bar- und Naturalrabatte an Apotheken, denen der Gesetzgeber schließlich den Riegel vorgeschoben hat, ebenfalls in großem Umfang zu Umstellungen beim Patienten kam - apothekeninduziert und aufgrund der Interessenlage gegenüber den Patienten sicherlich wohlwollender kommuniziert. Das WIdO hat gezeigt, dass es unter Rabattverträgen letztlich zu weniger Präparatewechseln beim Patienten kommt als vor der Durchsetzung der Rabattverträge. Untersucht wurden hierfür 32 Mio. wirkstoffbezogene Patientenprofile von 58 dauerhaft generikafähigen Wirkstoffen. Während im Jahr 2006 - vor Beginn der Rabattverträge - lediglich $70 \%$ der Patienten während des gesamten Jahres immer das gleiche Arzneimittel des jeweiligen Wirkstoffs erhalten hatten, lag dieser Anteil im Jahr 2010 mit 79 \% deutlich höher.

Nachdem es nicht zu einer Einigung über die Substitutionsliste gekommen war, hat der Gesundheitsausschuss die Vertragspartner in einem fraktionsübergreifenden Entschließungsantrag aufgefordert, die Liste bis August 2013 vorzulegen. Auch diese Frist verstrich

\section{Der Gesetzgeber hat für die Substitutionsentscheidung von Arzt und Apotheker im Einzelfall bereits solide Leitplanken geschaffen.}

ohne Ergebnis, da die Vertragspartner sich nicht auf die Kriterien für die Aufnahme von Arzneimitteln in eine Liste einigen konnten. In der Folge wollen die Koalitionspartner jetzt die Liste vom Gemeinsamen Bundesausschuss erarbeiten lassen.

Für die Delegation dieser Aufgabe an den Gemeinsamen Bundesausschuss spricht, dass die Aufnahme eines Wirkstoffs in die Liste mit erheblichen Konsequenzen für die Marktentwicklung dieses Wirkstoffs verbunden ist. Tendenziell ergibt sich hierdurch ein erheblicher Vorteil für den Originator, bzw. für ein bestehendes marktstarkes Generikaunternehmen mit zum Zeitpunkt der Listung hohem Marktanteil. Die Aufnahme eines Wirkstoffes muss daher nach gründlicher Prüfung des Sachverhaltes und unter klaren Kriterien in einem transparenten Verfahren getroffen werden. Der Gemeinsame Bundesausschuss ist im gleichen $\mathrm{Zu}$ sammenhang bereits mit der Substituierbarkeit von einzelnen Wirkstoffen und deren Darreichungsformen befasst und kann damit ein (auch für die Hersteller) transparentes widerspruchsfreies Verfahren besser sicherstellen als die bilateralen Verhandlungen der Vertragspartner.

\section{Ende der Salamitaktik: Abschaffung der Wirtschaftlichkeitsprüfung durch "regionale Vereinbarungen"}

Im Abschnitt „Ambulante Gesundheitsversorgung" haben sich die Koalitionspartner darauf geeinigt, „dass die heutigen Wirtschaftlichkeitsprüfungen bis Ende 2014 durch regionale Vereinbarungen von Krankenkassen und Kassenärztlicher Selbstverwaltung ersetzt werden“. Auffällig ist hier die kurze Frist, die Vertragspartnern gesetzt ist. Erfahrungsgemäß dauern Verhandlungen, in denen neue Verfahren zwischen Vertragspartnern mit durchaus unterschiedlichen Haltungen vereinbart werden sollen, ihre Zeit. Zeitdruck rächt sich häufig dadurch, dass bei der Umsetzung Probleme auftreten und die Akzeptanz der neuen Verfahren beschädigt wird. $\mathrm{Zu}$ beachten ist überdies, dass neue Formen von Zielvereinbarungen oder anderen Steuerungsmechanismen auch erhebliche datenlogistische Anforderungen stellen. Für eine wirksame Steuerung sollten Ärzte informiert sein, wie sie die vereinbarten Ziele in ihrer Praxis umgesetzt haben. Die Verhandlungen zur Konkretisierung des sog. ABDA/ KBV-Modells zeigen, wie viel Zeit solche Entwicklungsprozesse und ihre Umsetzung in der Datenlogistik in Anspruch nehmen.

Überdies ist unklar, warum die Koalitionspartner nach einer Kaskade von bereits erfolgten Abschwächungen insbesondere der Richtgrößenprüfung hier weiteren Handlungsbedarf gesehen ha- 
ben. Sie nehmen damit auch in Kauf, dass die Verordnungsbedingungen für die Vertragsärzte mehr als bisher regional differenziert werden. Aus Patientensicht erscheint dies wenig sinnvoll.

Der $\int 106$ SGB V, der die bisherigen Wirtschaftlichkeitsprüfungen regelt, ist in den letzten Jahren fast im Jahrestakt angepasst worden, entsprechend un-

\section{Wie sollen in Zukunft Ärzte motiviert werden, preisgünstige Wirkstoffe, z.B. Biosimilars, zu verordnen?}

übersichtlich stellt er sich mit nunmehr insgesamt 21 Absätzen dar. Schon diese Genese zeigt, dass es sich bei der Wirtschaftlichkeitsprüfung um umkämpftes Terrain handelt, von der umfangreichen Rechtsprechung hierzu ganz abgesehen. Die Vertragsärzte haben in den letzten Jahren immer wieder dafür plädiert, die Regressbedrohung für die Ärzte abzuschaffen - insbesondere vor dem Hintergrund des prognostizierten Ärztemangels wirke die Wirtschaftlichkeitsprüfung abschreckend auf die Bereitschaft zur Niederlassung. Der Gesetzgeber ist dieser Argumentation in den letzten Jahren vor allem dadurch gefolgt, dass bei einer erstmaligen Überschreitung der Richtgrößen zunächst eine Beratung zu erfolgen hat und ein Regress erst für den Zeitraum nach erfolgter Beratung möglich ist. In der Praxis ist ein Regress damit frühestens im dritten Jahr möglich. Zudem darf der erstmalig festgesetzte Regress 25.000 EUR nicht überschreiten.

Die Richtgrößenprüfung in ihrer derzeitigen erstarrten Form ist sicherlich nicht optimal. Der Gesetzgeber hat bereits mit dem AMNOG vorgesehen, dass die Vertragspartner auf Landesebene neue Prüfformen vereinbaren können und mit ihnen die Richtgrößenprüfungen ablösen können. Diese Neuregelung ist allerdings bisher nicht umgesetzt worden.

Die Kassenärztlichen Vereinigungen betrachten es überwiegend nicht als ihre Aufgabe, das Wirtschaftlichkeitsgebot unter ihren Mitgliedern durchzusetzen. Im Gegenteil: es kommt vor, dass sie sich in Einzelfällen durch ihr Agieren in den Gremien der Wirtschaftlichkeitsprüfung schützend auch vor das schwärzeste beratungsresistente Schaf stellen. Die Verhandlungen zur Weiterentwicklung der
Arznei- und Heilmittelvolumina und der Richtgrößen verlaufen in der Regel ritualisiert und ohne großen inhaltlichen Gestaltungsdrang. Die Frage ist daher, wie es bei dieser Interessensituation gelingen kann, dass durch regionale Verhandlungen tatsächlich eine effektive und angemessene Form der Prüfung entwickelt wird, die letztlich von den Ärzten und von den Krankenkassen akzeptiert wird. Wie sollen in Zukunft Ärzte motiviert werden, preisgünstige Wirkstoffe, z.B. Biosimilars, auszuwählen? Eine verbindliche Konkretisierung des Wirtschaftlichkeitsgebotes für den Arzt ist erforderlich. Wird gegen dieses Gebot in großem Umfang verstoßen, muss es auch in Zukunft Sanktionsmöglichkeiten geben. Die Erfahrungen haben gezeigt, dass allein durch positive Anreize (Bonus-Modelle) eine umfassende Verhaltenssteuerung nicht erreicht werden kann.

Die Neuregelung stellt nach den bisherigen Erfahrungen auf Landesebene ein Risiko dar, dass mit der Ablösung der derzeitigen Prüfverfahren ein Vakuum entsteht. Dies kann aus Kassensicht nicht akzeptiert werden. Ferner müssen die Auswirkungen einer Änderung des \$106 SGB V auch auf die Wirtschaftlichkeitsprüfungen anderer ambulanter Leistungserbringer (Hochschulambulanzen, psychiatrische Institutsambulanzen, die ambulante spezialfachärztliche Versorgung...) beachtet werden. Unter sinnvollen Rahmenbedingungen für die Verhandlungen besteht die Chance, tatsächlich zu einer angemessenen und beidseitig akzeptierten Form der Prüfung zu gelangen. Hier ist der Gesetzgeber gefordert, für beide Seiten der Verhandlung sinnvolle Leitplanken zu setzen, sodass regionale Blockaden vermieden werden.

\section{Ausweitung der Krankenhausverordnungen: Probleme an der Sektorengrenze}

Eine eher unauffällige Änderung findet sich im Abschnitt „Ambulante Gesundheitsversorgung": Im Kontext des Entlassungsmanagements sollen die Möglichkeiten der Krankenhäuser, bei einer Entlassung Leistungen zu verordnen, erweitert werden. Grundsätzlich spricht viel für einen möglichst reibungslosen Übergang von der stationären in die ambulante Versorgung. Die (schlechten) Erfahrungen mit den Regelungen des $\mathbb{1 1 5}$ c SGB V, wonach die Krankenhäuser gehalten sind, „bei der Entlassung Arzneimittel anzuwenden, die auch bei der Verordnung in der vertragsärztlichen Versorgung zweckmäßig und wirtschaftlich sind“, zeigen jedoch, dass die Krankenhausärzte die vertragsärztlichen Regularien für vertragsärztliche Arzneimittelverordnungen (Arzneimittelrichtlinien, regionale Arzneimittelvereinbarungen, Rabattverträge etc.) häufig nicht zur Kenntnis nehmen. Dies belastet auch die Vertragsärzte, die ihren Patienten erklären müssen, warum stationär und unterschiedlich verfahren wird. Bei einer Ausdehnung der Reichweite des Krankenhauses müssen diese Gesichtspunkte daher in den Blick genommen werden. Wichtiger als eine Ausdehnung der Krankenhausverordnungen wären Instrumente, die im Krankenhaus das Interesse an einer frühzeitigen Absprache mit den behandelnden Vertragsärzten wecken.

\section{Ausblick}

Die große Koalition hat sich noch 2013 als handlungsfähig erwiesen und die wichtigsten Regelungen auf den Weg gebracht. Auch die das AMNOG-Verfahren betreffenden Regelungen sollten unverzüglich umgesetzt werden, um Rechtssicherheit herzustellen. Mehr Bedenk- und Verhandlungszeit für die Partner bei der Fortentwicklung der Wirtschaftlichkeitsprüfung wäre sinnvoll. 